Von diesen Säuren ist die erste, wie schon erörtert, $\alpha$-Methyl- $\alpha$-carbonsäure :

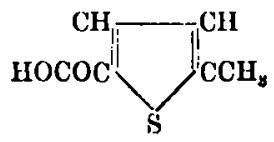

denn sie wurde von $\mathrm{Pa}$ al aus einem $\alpha \alpha$-Dimethylthiophen gewonnen.

Der zweiten und dritten müssen der Entstehung nach die Formeln :

II.<smiles>CC1(C)CSC1C(=O)O</smiles>

und

III.<smiles>C[C@@H]1[SiH2]CC(C(=O)O)[C@H]1C</smiles>

zukomınen.

Vorstehende Untersuchung wurde im Laboratorium des chemischen Instituts der Universität Halle-Wittenberg auf Veranlassung und unter Leitung des Herrn Prof. Volhard ausgeführt.

Ueber Verbindungen des Thiophens, seiner Homologen und einiger Ketone mit Quecksilberchlorid; von $J$. Volhard.

In dem bis dabin bekannt gewordenen Verhalten des Thiophens und seiner Derivate macht sich der Schwefelgehalt dieser Körper, aufser bei ihrer vollständigen Zerstörung durch Oxydationsmittel, sonderbarer Weise gar nicht bemerklich. Das Schwefelatom führt vielmehr die einmal übernommene Rolle des Vertreters für ein Acetylen des Benzols unter den verschiedensten Umständen beharrlich durch. Um so mehr dürften die nachstehend beschriebenen Verbindungen interes- 
siren, als sie die ersten sind, in deren Bildung sich das Thiophen wie die Schwefelverbindung eines organischen Radicals verhält. Thiophen und seine Homologen bilden nämlich mit Quecksilberchlorid Verbindungen, wie man solche von den Schwefelalkylen, vom Allyl- und Vinylsulfid kennt.

Schüttelt man Thiophen oder eines seiner Homologen mit einer wässerigen Sublimatlösung, so umkleiden sich die 0eltröpfchen allmählich mit einer weirsen Haut, und nach einiger Zeit fällt eine Verbindung, welche die Elemente des Thiophens, Quecksilber und Chlor enthält, als weifser voluminöser Niederschlag aus, während die Flüssigkeit stark saure Reaction annimmt. Der Niederschlag ballt zu Klümpchen zusammen, welche im Innern Thiophen einschliefsen und der Berührung mit der Quecksilberlösung entziehen; andererseits wird durch die freiwerdende Salzsäure, da diese die Thiophenquecksilberverbindungen zersetzt, verhindert, dafs alles Thiophen mit dem Sublimat in Reaction trete. Man vermeidet das Klumpigwerden des Niederschlags, indem man das Thiophen mit Weingeist verdünnt, und die freiwerdende Salzsäure bindet man durch essigsaures Natron. Gleichwohl gelingt es nicht alles Thiophen niederzuschlagen, und die Versuche, aus den Theerölen die Thiophene mit Quecksilberchlorid abzuscheiden, haben mir höchst unbefriedigende Ergebnisse geliefert.

Die Bildung dieser Quecksilberverbindungen aus dem Thiophen und seinen Homologen erfolgt in der Weise, dafs Wasserstoff aus dem Thiophen mit der Hälfte des Chlors im Sublimat Chlorwasserstoff bildet, während der Rest des Quecksilberchlorids $\mathrm{HgCl}$ an die Stelle von Wasserstoff in das Thiophen eintritt.

In der Regel entstehen gleichzeitig zwei Verbindungen, indem entweder ein oder zwei Atome Wasserstoff des Thiophens durch $\mathrm{HgCl}$ ersetzt werden. Die Monoquecksilberchloridverbindungen sind in Wasser etwas, wenn auch wenig, 
auflöslich, reichlicher in kochenden Weingeist, aus welchem man sie gut umkrystallisiren kann, während die Diquecksilberverbindungen in allen indifferenten Lösungsmitteln ganz unlöslich sind.

Durch Salzsäure werden diese Quecksilberverbindungen zersetzt unter Rückbildung des zu ihrer Darstellung angewendeten Thiophens, daneben entsteht Quecksilberchlorid. Eine Abscheidung von Quecksilbersulfid oder Bildung flüchtiger organischer Chlorverbindungen, wie sie bei der Zersetzung der Verbindungen aus Allyl- oder Vinylsulfid und Sublimat von Semmler*) nachgewiesen wurde, konnte in keinem Falle beobachtet werden. Die Zersetzung mit Salzsäure erfolgt bei den Monoquecksilberderivuten leicht, während die Diverbindungen nur durch ganz concentrirte Säure, bei anhaltender Digestion in der Wärme und selbst dann in der Regel nicht vollständig in ihre Componenten aufgespalten werden.

Chlorverbindungen der Radicale einbasischer Säuren geben mit den Monoverbindungen sehr leicht Quecksilberchlorid und die Thienylketone, welche V. Meyer mittelst der FriedelCrafts'schen Reaction dargestellt und als Thienone bezeichnet hat.

Einige dieser Ketone sind von Dr. A. Thiele analysirt worden; ihre Identität mit den V. M e yer'schen Thienonen beweist, dafs es die $\alpha-W$ asserstoffatome des Thiophens sind, welche durch $\mathrm{HgCl}$ substituirt werden.

Mlit den Diquecksilberverbindungen Diketone zu erzeugen ist mir nicht gelungen.

Chlorverbindungen der Radicale zweibasischer Sauren, wie Succinylchlorid, Chlorkohlenoxyd wirken aul die Quecksilberverbindungen nicht ein; ebensowenig die Ester halogensubstituirter Säuren oder Halogenverbindungen von Alhylen.

*) Lieso Annalen 241, 121. 
Auch flüssiges Chlorcyan mit der Monoquecksilberverbindung im zugeschmolzenen Rohr erhitzt, blieb ohne Einwirkung.

Zur Bestimmung des Quecksilbers wurden die Monoverbindungen in kochender weingeistiger Lösung mit Schwefelwasserstoff zersetzt und bis zum Absitzen des Quecksilbersulfids in der Wärme digerirt; im Filtrat fält man das Chlor durch Silberlösung. Zur Bestimmung des Schwefels wurde die Substanz mit concentrirter Salpetersäure zuerst im offenen Rohr gelinde erwärmt; nach Beendigung der heftigen Reaction wurde das Rohr zugeschmolzen und 7 bis 8 Stunden lang bei 200 bis $230^{\circ}$ erhitzt; der Rohrinhalt wurde dann in Wasser aufgenomaen, mit reinem Natron übersättigt, in einer Silberschale eingedampft, geschmolzen und zuletzt bis zum ruhigen Fliefsen erhitzt. Nach Verjagung der Salpetersäure durch wiederholtes Abdampfen mit Salzsäure fällte man mit Chlorbaryum.

Die Diverbindungen setzten der Analyse einige Schwierigkeiten entgegen. Um sie mit Salzsäure zu zersetzen, mufs man so anhaltend erhitzen, dafs Verlust von Quecksilber zu befürchten ist, auch ist die Zersetzung in der Regel nicht ganz vollständig. Schwefelammonium zersetzt nur oberflächlich ; beim Erhitzen mit Schwefelammonium im zugeschmolzenen Rohr bei 150 bis $160^{\circ}$ platzten alle Röhren nach 3 bis 4 Stunden, bevor die Substanz völlig zersetzt war. Unvollständig bleibt auch die Zersetzung mit Schiwefelammonium und Natronlauge, insofern dabei bald mehr bald weniger, immer freilich nur sehr wenig von der Substanz in grauen Flocken ungelöst zurückbleibt. Nach vielen Versuchen blieb man bei folgendem Verfahren der Quecksilberbestimmung stehen : Die Substanz wurde mit verdünnter Salpetersäure im geschlossenen Rohr 6 bis 8 Stunden lang bei $100^{\circ}$ erhitat; sie geht dabei nur zum kleinen Theil in Lösung, aher der Rückstand ist derartig 
aufgelockert, dafs nunmehr beim Kochen mit Natronlauge und Schwefelammonium völlig klare Lōsung erfolgt. Das Quecksilber wird jetzt durch Zusatz von Ammoniumnitrat und anhaltende Digestion in der Wãrme zur Abscheidung gebracht; das Filtrat vom Schwefelquecksilber wurde zur Trockene verdampft; nach Zusatz von Salpeter geschmolzen und aus der Lösung der Schmelze das Chlor mit Silbernitrat gefällt. Häufiger wurde zur Bestimmung von Schwefel und Chlor die Substanz mit dem vierzigfachen Gewicht eines Gemenges von kohlensaurem Natron (1 Th.) und Salpeter (2 Th.) innig gemischt in geschmolzenes Aetznatron eingetragen; die Lösung der Schmelze wurde getheilt; ein Theil diente zur Chlor-, der andere zur Schwefelbestinmung. Zur Elementaranalyse verbrannte man die Substanz nit Bleichromat; vor die Mischung kam eine lange Schicht gekörntes Chromat, welche während der Verbrennung nur mäfsig erhitzt wurde. Der vordere aus dem Ofen herausragende Theil des Bohres war mehrfach verengt; er wurde nach der Verbrennung abgeschmolzen und das Wasser mit der Luftpumpe in das (hlorcalciumrohr übergetrieben *). Das Verbrennungsrohr mufs schr lang genommen und die Verbrennung sehr langsam geführt werden, sonst geht leicht bis in das Chlorcalciumrohr ein krystallinisches Sublimat über, welches aus Quecksilberchlorid besteht.

Monoquecksilberchloridthiophen, $\mathrm{C}_{4} \mathrm{H}_{9} \mathrm{SHgCl}$. - In eine grofse Flasche giebt man $10 \mathrm{Th}$. Thiophen, $100 \mathrm{Th}$. Weingeist, 1000 Th. einer kalt gesättigten Sublimatlōsung und 200 Th. einer Lösung von essigsaurem Natron, welche etwa ${ }^{1_{1}}$ ihres Gewichtes krystallisirtes Salz enthält. Man schüttelt tüchtig durch und läfst unter öfterem Schütteln 4 bis 5 Tage stehen. Der weifse krystallinische Niederschlag wird ahfiltrirt. Das Filtrat läist in der Regel bei längerem Stehen noch

*) Frankland und Duppa, diese Annalen 180 , 115 Anm. 
ziemlich viel des gleichen Niederschlages ansfallen. Nach dem Auswaschen mit kaltem Wasser kocht man den Niederschlag wiederholt mit reichlichen Mengen von Weingeist aus, so lange die Lösung beim Erkalten noch Krystalle abscheidet. Man filtrirt mit dem Heifswassertrichter. Die Quecksilberverbindung krystallisirt beim Erkalten in weifsen Blättchen, welche in der Flüssigkeit fast wie blankes Silber glänzen und nach dem Trocknen ein weifses abfärbendes Pulver darstellen. In kaltem Wasser ist der Körper fast unlöslich; aus kochendem, von welchem er auch nicht eben reichlich aufgenommen wird, fältt er beim Erkalten als krystallinisches Pulver aus. Er schmilzt bei $183^{\circ}$ und ist etwas flüchtig; die Sublimation beginnt wenig über $100^{\circ}$ und wird bei $210^{\circ}$ lebhaft; es lassen sich jedoch nur kleine Mengen sublimiren, in grö[seren Mengen erhitzt, zersetzt sich der Körper unter Schwärzung.

1. $0,1880 \mathrm{~g}$ gaben $0,1356 \mathrm{Hg}$ s entspr. $0,1169 \mathrm{Hg}$ and $0,0878 \mathrm{AgCl}$ entspr. $0,0217 \mathrm{Cl}$.

2. $0,3312 \mathrm{~g}$ gabeu $0,2385 \mathrm{HgS}$ entspr. $0,2056^{\circ} \mathrm{Hg}$ und $0,1502 \mathrm{AgCl}$ entspr. $0,03758 \mathrm{Cl}$.

3. 0,6546 g gaben $0,4683 \mathrm{BaSO}_{4}$ entspr. $0,0659 \mathrm{~S}$.

4. $0,6361 \mathrm{~g} n 0,4630$ n 0,0643 n

5. $0,7960 \mathrm{~g} n\left(\mathrm{CO}\right.$, verungliickt) $0,0866 \mathrm{H}_{\mathbf{2}} \mathrm{O}$ entspr. $0,0096 \mathrm{H}$.

b. $0,5288 \mathrm{~g}, 0,2863 \mathrm{CO}_{2}$ entepr, $0,07808 \mathrm{C}$ und $0,0586 \mathrm{H}_{2} \mathrm{O}$ entspr. $0,0065 \mathrm{H}$.

Berechnet für

$\mathrm{C}_{4} \mathrm{H}_{3} \mathrm{SHgCl}$

Hg

Cl

$\mathbf{S}$

H

c
62,80

11,14

10,05

0,94

15,07

\begin{tabular}{cccc}
\multicolumn{4}{c}{ Gefunden } \\
\hline 1. u. 3. & 2. u. 4. & 5. & b. \\
62,18 & 62,08 & - & - \\
11,55 & 11,35 & - & - \\
10,07 & 10,11 & - & - \\
- & - & 1,20 & 1,23 \\
- & - & - & 14,77
\end{tabular}

Diquecksilberchloridthiophen, $\mathrm{C}_{4} \mathrm{H}_{2} \mathrm{SHg}_{2} \mathrm{Cl}_{2}$, ist ein weifses abfärbendes erdiges Pulver, in allen indifferenten Lösungsmitteln unlöslich; es wird von Sãuren sehr schwer angegriffen, 
selbst von concentrirter Salpetersäure; wässerige Alkalien sind ohne Einwirkung auf dasselbe.

1. 0,5604 g gaben $0,4729 \mathrm{HgS}$ ontspr. $0,4077 \mathrm{Hg}$ und $0,2955 \mathrm{AgCl}$ entspr. $0,0731 \mathrm{Cl}$.

2. $0,3568 \mathrm{~g}$ gaben $0,2990 \mathrm{HgS}$ entspr. $0,2577 \mathrm{Hg}$.

3. $0,6443 \mathrm{~g} \rightarrow$ je zur Halfte $0,1650 \mathrm{AgCl}$ entspr. 0,04082 Cl

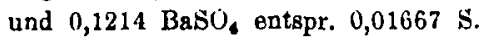

4. $0,39245 \mathrm{~g}$ gaben $0,2033 \mathrm{AgCl}$.

5. 0,7416 g $, 0,2439 \mathrm{CO}_{2}$ entspr. $0,0665 \mathrm{C}$ und $0,0340 \mathrm{H}_{2} \mathrm{O}$ entspr. $0,00378 \mathrm{H}$.

b. $0,8428 \mathrm{~g}$ gaben $0,2757 \mathrm{CO}_{2}$ entspr. $0,0752 \mathrm{C}$ und $0,0262 \mathrm{H}_{8} \mathrm{O}$ entspr. 0,00294 $\mathrm{H}$.

Berechnet für $\mathrm{C}_{4} \mathrm{H}_{2} \mathrm{SH}_{8} \mathrm{Cl}_{2}$

$\begin{array}{lr}\mathrm{H}_{\mathbf{g}} & 72,32 \\ \mathrm{Cl} & 12,84 \\ \mathrm{~S} & 5,80 \\ \mathrm{H} & 0,36 \\ \mathrm{C} & 8,68 \\ & . . . \\ & 100,00 .\end{array}$

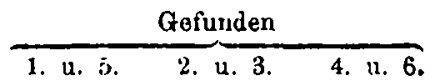

Acetothienon oder Thienymethylketon *), $\mathrm{CH}_{\mathbf{3}} \mathrm{COC}_{3} \mathrm{H}_{3} \mathrm{~S}$.

$10 \mathrm{~g}$ Monoquecksilberchloridthiophen wurden mit $3,5 \mathrm{~g}$ Acelylchlorid in Wasserbade am Rückflufskühler kurze Zeit schwach erwärmt. Gleich im Beginn der Reaction färbte sich die Masse fleischroth, dann allmählich schwarz. Nach $\forall$ stündigem Stehen war der Geruch nach Acetylchlorid verschwunden, und ein aromatischer Geruch zu bemerken. Auf Zusatz von kohlensaurem Natron bis zur alkalischen Reaction fiel das Quecksilber als gelbes Quecksilberoxyd uus. Nun wurde mit Aether ausgeschüttelt. Das nach dem Abdussten des Aethers hinterbliebene dunkelbraune Oel wurde fractionirt. Bei 212

*) Die weiteren Versuche siud mehrentleils rou 1)r. A. Thiele ausgefïhrt. Vgl, desseu Dissertation, llalle 1891. 
seiner Homologen u. einiger Ketone mit Quecksilberchlorid. 179

bis $213^{\circ}$ ging ein hellgelb gefarbtes, aromatisch riechendes Oel uber, das bei längerem Stehen eine dunklere Färbung annahm. Die Ausbente betrug $77 \mathrm{pC}$. der nach der Gleichung $\mathrm{C}_{4} \mathrm{H}_{3} \mathrm{SHgCl}+\mathrm{CH}_{3} \mathrm{COCl}=\mathrm{C}_{4} \mathrm{H}_{3} \mathrm{SCOCH}_{3}+\mathrm{HgCl}_{3}$ berechneten Menge.

Das so dargestellte Oel ist identisch mit dem von Peter*) aus dem Thiophen nach dem Fried el-Crafts'schen Verfahren vermittelst Aluminiumchlorid erhaltenen Acetothienon, dessen Siedepunkt zu 213,50 angegeben wird.

Lin dasselbe in Oxim überzuführen wurden $2 \mathrm{~g}$ des Reactionsproductes mit $2,2 \mathrm{~g}$ salzsaurem llydroxylamin und der berechneten Menge Soda in alkoholischer Lösung am Rückflufskühler auf dem Wasserbade 12 Stunden lang erwarmt. Die alkoholische Lösung wurde in Wasser gegossen, worauf nach einigen Tagen die Abscheidung eines Krystallbreies erfolgte. Durch Umkrystallisiren aus heifsen Wasser erhält mun das Oxim als weifse Krystallmasse von Schmelzpunkt 110 bis $112^{\circ}$, Peter giebt den Schmeizpunkt 110" an.

0,1734 g Substanz gaben $0,3215 \mathrm{CO}_{2}$ entspr. $0,0877 \mathrm{C}$ und 0,0783 $\mathrm{H}_{3} \mathrm{O}$ entspr. $0,0087 \mathrm{H}$.

0,1213 g gaben 0,196 BaSO, entspr. 0,027 S.

$\begin{array}{ccc} & \text { Berechuet für } & \text { Gefunden } \\ & \mathrm{C}_{\mathbf{4}} \mathrm{H}_{8} \mathrm{SC}(\mathrm{NOH}) \mathrm{CH}, & \\ \text { C. } & 51,06 & 50,58 \\ \text { H } & 4,96 & 5,00 \\ \text { S } & 22,7 & 22,26 .\end{array}$

Beim mäfsigem Erhitzen mit Phenylhydrazin entstand die Hydrazinverbindung : gelbe, leicht zersetzliche Nadeln, welche den auch von $P$ eter beobachteten Schnelzpunkt $96^{\circ}$ zeigen.

$$
\text { Thienylphenylketon, } \mathrm{C}_{6} \mathrm{H}_{5} \mathrm{COC}_{4} \mathrm{H}_{5} \mathrm{~S} \text {. }
$$

$10 \mathrm{~g}$ Monoquecksilberchloridthiophen wurden mit $4,4 \mathrm{~g}$ Benzoylchlorid gemischt und im Wasserbade am Rückflurs-

*) Ber. d. deutsch. chem. Ges. 1 z, 2t343. 
kühler kurze Zeit erwärmt. Auch hier färbte sich die Masse anfangs fleischroth, dann schwarz. Kohlensaures Natron schied ein braunes Oel ab, welches mit Wasserdämpfen übergetrieben wurde. Die ätherische Lõsung hinterliefs eine feste, krystallinische Masse, die aus Alkohol umkrystallisirt schöne farblose Nadeln vom Schmelzpunkt $56^{\circ}$ lieferte. Co$m$ ey ${ }^{*}$ ) giebt den Schmelzpunkt des vermittelst Aluminiumchlorid dargestellten Phenylthienylketons $2 u 5^{\circ}$ an.

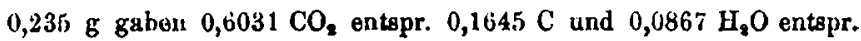
$0,0096 \mathrm{HI}$.

(1,3273 g galuen 0,4121 BaSO, entspr. 0,0666 s.

$\begin{array}{ccc} & \text { Berechnet für } & \text { Gefunden } \\ \text { C } & \mathrm{C}_{4} \mathrm{H}_{8} \mathrm{SCOC}_{6} \mathrm{H}_{8} & \\ \text { H } & 70,2 & 70 \\ \text { S } & 4,25 & 4,1 \\ & 17,1 & 17,28 .\end{array}$

Dijodthiophen. - Jod wirkt auf die Monoquecksilberverbindung nicht ein, dagegen verwandelt es die Diquecksilberverbindung leicht in Dijodthiophen, wenn es in der berechneten Menge damit unter Zusatz von Wasser erwärmt wird. Das l'roduct wurde mit Wasser übergetrieben, in Aether aufgenommen und nach dessen Verdampfen aus Alkohol umkrystallisirt, aus welchem es nach eiuiger Zeit in schönen weifsen Blättchen vom Schmelzpunkt $40^{\circ}$ herauskam.

$0,2314 \mathrm{~g}$ gaben $0,3215 \mathrm{AgJ}$ entspr. $0,1738 \mathrm{~J}$ oder $75,1 \mathrm{pC}$. Berechnet für $\mathrm{C}_{4} \mathrm{H}_{\mathbf{g}} \mathrm{J}_{2} \mathrm{~S} 75,6 \mathrm{pC}$.

$\alpha$-Methylthiophenquecksilberchlorid.

(q-Methylthiophen, dargestellt nach P a a $\left.{ }^{* *}\right)$ durch Destillation von 1 Th. Lävulinsäure und 11/2 Th. Phosphortrisulfid, liefert mit Quecksilberchlorid und essigsaurem Natron fasl nur die Monoquecksilberverbindung. Der weifse flockige Nieder-

$\because$ lier. d. deutsch. chem. Ges. 18, 7su.

**) Daselbst $19, \mathbf{5 . 5 6}$. 
schlag, der sich bei längerem Stehen der Mischung und hüufigem Umschütteln bedeutend vermehrte, wurde von kochendem Alkohol fast ganz gelöst; er wurde aus Alkohol umkrystallisirt. Kurze, durchsichtige Nadeln, meistentheils auf beiden Seiten wetzsteinartig zugespitzt; unlöslich in Wasser und Aether; Schmelzpunkt 197. Iie Ausbeute betrug 44 pC. der Theorie.

1. $0,7627 \mathrm{~g}$ gaben 0,5594 $\mathrm{BaSO}_{4}$ entspr. 0,0768 S.

2. $0,0963 \mathrm{~g}, 0,067 \mathrm{HgS}$ entspr. $0,0578 \mathrm{Hg}$ und $0,0420 \mathrm{AgCl}$ entspr. 0,0104 Cl.

3. $0,3250 \mathrm{~g}$ gaben $0,217 \mathrm{CO}$, entspr. $0,0592 \mathrm{C}$ und $0,0436 \mathrm{H}_{8} \mathrm{O}$ entspr. $0,00484 \mathrm{H}$.

4. $0,3934 \mathrm{~g}$ gaben $0,2647 \mathrm{CO}_{2}$ entspr. 0,0722 $\mathrm{C}$ und $0,0548 \mathrm{H}_{3}$ () entspr. $0,0061 \mathrm{II}$.

$\begin{array}{lcc} & \text { Berechnet für } & \text { Gefunden } \\ & \mathrm{C}_{\triangleleft} \mathrm{H}_{2} \mathrm{CH}_{8} \mathrm{SH}_{\mathrm{gCl}} & \\ \mathrm{S} & 9,62 & 10,07 \\ \mathrm{Hg} & 60,15 & 59,98 \\ \mathrm{Cl} & 10,68 & 10,79 \\ \mathrm{C} & 18,1 & 18,2 \\ \text { H } & 1,5 & 1,5 .\end{array}$

\section{Phenylthiotolylketon, $\mathrm{C}_{6} \mathrm{H}_{5} \mathrm{COC}_{4} \mathrm{H}_{2} \mathrm{SCH}_{3}$.}

3 g a-Methylthiophenquecksilberchlorid wurden längere Zeit mit 1,3 g Benzoylchlorid auf dem Wasserbade erwärmt. Mit kohlensaurem Alkali stark alkalisch gemacht, wurde das Reactionsproduct mit Aether aufgenommen. Das ausgeschiedene gelbe Quecksilberoxyd wurde von der ätherischen Lösung getrennt, letztere getrocknet, und der Aether bis auf einen kleinen Rest abdestillirt. Nach freiwilligem Verdunsten des Aethers blieb ein dunkelgefärbtes Oel zurück, das beim längeren Stehen im Exsiccator krystallinisch erstarrte.

Die Krystallmasse liefs sich durch Abpressen und Umkrystallisiren aus Ligroin leicht reinigen. 
Abweichend von den Angaben Ernst's *), welcher das aus Theerthiotolen mittelst Aluminiumchlorid dargestellte Keton als ein Oel beschreibt, erhielt ich schöne, silberglänzende Krystallnadeln vom Schmelzpunkt $124^{\circ}$.

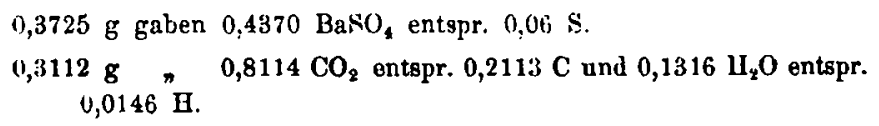

$\begin{array}{ccc} & \begin{array}{c}\text { Berochnet für } \\ \mathrm{C}_{6} \mathrm{H}_{8}\left(\mathrm{OC}_{4} \mathrm{U}_{9} \mathrm{SCH}\right.\end{array} & \text { Ciotundon } \\ \text { S } & 15,84 & 16,1 \\ \text { C } & 71,28 & 71,1 \\ \text { H } & 4,95 & 4,69 .\end{array}$

$\beta$-Thiotolenquecksilberchlorid.

Thiotolen aus Brenzweinsãure wurde ganz ebenso wie oben für Thiophen beschrieben mit Quecksilberchlorid behandelt.

Monoquecksilberchlorid- $\beta$-thiotolen, $\mathrm{C}_{5} \mathrm{H}_{5} \mathrm{~S} . \mathrm{HgCl}$, krystallisirt aus der heifsen Lōsung in absolutem Alkohol beim Erkalten in feinen weifsen, büschelförmig vereinigten Nädelchen ; viel davon bleibt in der alkoholischen Mlutterlauge gclöst und läfst sich daraus durch Verdünnen mit $W$ asser abscheiden.

$0,4207 \mathrm{~g}$ galuen $0,2976 \mathrm{llgs}$ entspr. $0,2565 \mathrm{Hg}$ und $0,1804 \mathrm{AgCl}$ entspr. 0,(1446 Cl.

$\begin{array}{lcc} & \text { Berechnet für } & \text { Gofunden } \\ \mathrm{Hg} & \mathrm{C}_{5} \mathrm{H}_{8} \mathrm{SHgCl} & \\ \mathrm{Cl} & 60,18 & 60,97 \\ & 10,65 & 10,60 .\end{array}$

Diquecksilberchlorid- $\beta$-thiotolen, $\mathrm{C}_{5} \mathrm{H}_{4} \mathrm{~S} . \mathrm{Hg}_{2} \mathrm{Cl}_{2}$, ist weifs, körnig, in allen Lösungsmilteln unlōslich und wird selbst von concentrirter Salpetersäure nur schwer angegriffen.

0,4566 g gaben 0,1929 $13 \mathrm{aSO}$, entspr. 0,0265 $\mathrm{s}$.

$0,2102 \mathrm{~g} n \quad 0,1714 \mathrm{HgS}$ entspr. 0,1477 Hg.

$0,2102 \mathrm{~g} \cap 0,1055 \mathrm{AgCl} \cap 0,0261 \mathrm{Cl}$.

*) Ber. d. deutsch. chem. Ges. 19, 3280. 
seiner Homologen u. einiger Ketone mit Quecksilherchlorid. 183

$\begin{array}{lcc} & \text { Berechnet für } & \text { Gefunden } \\ & \mathrm{CC}_{4} \mathrm{HSCH}_{3} \mathrm{Hg}_{2} \mathrm{Cl}_{\mathbf{2}} & \\ \text { s } & \mathbf{5 , 6 4} & 5,8 \\ \mathrm{Hg} & 70,54 & 70,26 \\ \mathrm{Cl} & 12,52 & 12,41 .\end{array}$

B-Isopropylthiophenquecksilherchlorid.

$5 \mathrm{~g} \beta$-Isupropylthiophen dargestellt aus Isopropylbernsteinsäure oder Pimelinsäure gaben $15 \mathrm{~g}$ Quecksilberverbindung, ein Gemisch von $10 \mathrm{~g}$ der in Alkulıol löslichen Mono- und $5 \mathrm{~g}$ der in Alkohol unlöslichen Diverbindung.

Monoquecksilberchloridisopropylthiophen, $\mathrm{C}_{3} \mathrm{H}_{7} \cdot \mathrm{C}_{4} \mathrm{H}_{2} \cdot \mathrm{HgCl}$, krystallisirt aus heifsem Alkohol in schönen, langen weifsen Nadeln; es is: auch in Aether löslich, nicht in Wasser; Schmelzpunkt $137^{\circ}$.

1. $0,1020 \mathrm{~g}$ gaben $0,066 \mathrm{Hg}$ s entsprr. $0,0568 \mathrm{Hg}$ und $0,042 \mathrm{AgCl}$ entspr. $0,0103 \mathrm{Cl}$.

2. $0,2778 \mathrm{~g}$ gaben $0,2354 \mathrm{CO}_{2}$ entspr. $0,0642 \mathrm{C}$ und $0,071 \mathrm{H}_{2} \mathrm{O}$ entspr. $0,0079 \mathrm{H}$.

3. $0,2175 \mathrm{~g}$ gaben $0,1847 \mathrm{CO}_{3}$ entspr. $\mathrm{U}, 0504 \mathrm{C}$ und $0,0522 \mathrm{H}_{8} \mathrm{O}$ entspr. $0,0058 \mathrm{H}$.

Berechnet für $\mathrm{C}_{4} \mathrm{H}_{2}\left(\mathrm{C}_{8} \mathrm{H}_{7}\right) \mathrm{SHgCl}$

$\mathrm{Hg}$

55,48

Cl

9,85

C $\quad 23,3$

$\mathrm{H} \quad 2,5$

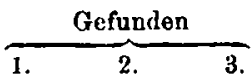

Diquecksilberchloridisopropylthiophen, $\mathrm{C}_{3} \mathrm{H}_{7} . \mathrm{C}_{4} \mathrm{HS}_{2} \mathrm{Hg}_{2} \mathrm{Cl}_{9}$, ist eine weifse, körnige Masse, unlöslich in allen Lösungsmitteln und wird von concentrirter Salpetersäure nur schwer angegriffen.

1. $0,0971 \mathrm{~g}$ gaben $0,0756 \mathrm{HgS}$ entspr. $0,0652 \mathrm{H}$ und $0,0465 \mathrm{AgCl}$ entspr. $0,0115 \mathrm{Cl}$.

2. $0,3325 \mathrm{~g}$ gaben $0,1705 \mathrm{CO}_{2}$ entspr. $0,0465 \mathrm{C}$ und $0,0449 \mathrm{H}_{3} \mathrm{O}$ entspr. $0,003 \mathrm{HI}$.

3. $0,3791 \mathrm{~g}$ gaben $0,138 \mathrm{BaSO}_{4}$ entspr. 11,019 s. 


\begin{tabular}{lcccc} 
& $\begin{array}{c}\text { Berechnet fiii } \\
\mathrm{C}_{4} \mathrm{HSG}_{2} \mathrm{H}_{\mathbf{7}} \mathrm{Hg}_{2} \mathrm{Cl}_{2}\end{array}$ & \multicolumn{3}{c}{ Gefunden } \\
$\mathrm{Hg}$ & 67,23 & 67,14 & - & - \\
$\mathrm{CI}$ & 11,93 & 11,82 & - & - \\
$\mathrm{C}$ & 14,1 & - & 13,98 & - \\
$\mathrm{H}$ & 1,34 & - & 1,5 & - \\
$\mathrm{S}$ & 5,4 & - & - & $5,00$.
\end{tabular}

Quecksilberchloridverbindungen der Ketone.

Nach den vorstehend beschriebenen Beobachtungen lag die Frage nahe, ob auch die acetylirten Thiophene in derselben Weise wie die alkylirten befähigt seien, Quecksilberchloridverbindungen zu liefern.

Diesbezügliche Versuche haben ergeben, dafs das 'Thienon sich allerdings mit Quecksilberchlorid verbindet, aber nicht wie das Thiophen und seine Homologen unter Austausch von Wasserstoff gegen cinen Rest des Quecksilberchlorids, sondern additionell, ohne Austritt von Chlorwasserstoff.

Merkwürdiger Weise bilden auch andere schwefelfreie Ketone solche molecularen Verbindungen mit Quecksilberchlorid, so das Acetophenon und Benzophenon.

2 g Acetothienon, in wenig Alkuhol gelöst, wurden mit einer kalt gesättigten Lösung von $9,34 \mathrm{~g}$ Sublimat und wenig essigsaurem Natron versetzt. Die milchige Lösung wurde schwach angewärmt, filtrirt und in eine flache Schale gegossen. Nach einiger Zeit schieden sich lange, weilse Nadeln aus, die durch Uınkrystallisiren aus Aether gereinigt wurden. Dieselben zeigen den Schmelzpunkt $68^{\circ}$ und lassen deutlich den Ketongeruch wahrnehmen.

$0,2615 \mathrm{~g}$ Substan\% gaben 0,1572 Hgs entspr. 0,1355 Hit und $0,1988 \mathrm{AgCl}$ entspr. 0,(1492 Cl.

$\begin{array}{lcc} & \text { Berechuet lïr } & \text { Getunden } \\ \mathrm{Hg} & \mathrm{C}_{2} \mathrm{H}_{3} \mathrm{OC} \mathbf{H}_{8} \mathrm{~N} . \mathrm{HgCl}_{2} & \\ \text { (l) } & 50,4 & 51,8 \\ & 17,9 & 18,8 .\end{array}$


seiner Homologen u. einiger Kétone mit Quecksilberchlorid. 185

Ebenso wie Acetothienon verhalten sich die Ketone, Acetophenon und Benzophenon; zur Bildung dieser Doppelverbindungen ist mithin die Anwesenheit von Schwefel nicht erforderlich.

$2 \mathrm{~g}$ Acetophenon, in wenig Alkohol gelöst, wurden mit der zur Bildung der Doppelverbindung nothwendigen Menge Quecksilberchlorid versetzt und ganz ebenso behandeit wie gerade vom Acetothienon beschrieben ist; nach einiger Zeit schieden sich grofse, weifse Nadeln ab. Dieselben sind löslich in Wasser, Alkohol und Aether und schmelzen bei $59^{\circ}$.

$0,4420 \mathrm{~g}$ gaben $0,2624 \mathrm{HgS}$ entspr. $0,2262 \mathrm{Hg}$ und $0,1167 \mathrm{AgCl}$ entopr. $0,0289 \mathrm{Cl}$.

$\begin{array}{lcc} & \text { Berechnet für } & \text { Gefunden } \\ \mathrm{C}_{6} \mathrm{H}_{6} \mathrm{COCH}_{3} \mathrm{HgCl} & \\ \mathrm{H} & 51,14 & 51,18 \\ \mathrm{Cl} & 18,16 & 18,3 .\end{array}$

Bei der Darstellung der Doppelverbindung aus Benzophenon und Quecksilberchlorid wurde ganz in derselben Weise verfahren, wie bei den schon angeführten. Lange, weifse, in Wasser, Alkohol und Acther leicht lösliche Nadeln vom Schmelzpunkt $\mathbf{8 1}^{\circ}$.

0,1906 g Substany gabes $0,1003 \mathrm{Hgs}$ entspr. $0,08647 \mathrm{Hg}$ und $0,1227 \mathrm{AgCl}$ entspr. 0,0305 Cl.

$\begin{array}{ccc} & \begin{array}{c}\text { Berechnet für } \\ \mathrm{C}_{6} \mathrm{H}_{5} \mathrm{COC}_{6} \mathrm{H}_{5} \mathrm{H}_{\mathrm{g}} \mathrm{Cl}_{2}\end{array} & \text { Gefunden } \\ \mathrm{Hg} & 45,25 & 45,3 \\ \mathrm{Cl} & 15,5 & 15,9 .\end{array}$

Alle diese Verbindungen sind sehr leicht zersetzlich; sie riechen immer nach dem in ihnen enthaltenen Keton, welches sich allmählich verflüchtigt, wenn die Verbindung an der Luft steht. Die Analysen ergaben daher immer einen kleinen U'eberschufs von Quec:. silberchlorid. 